\title{
ORLICZ SPACES AND NONLINEAR ELLIPTIC EIGENVALUE PROBLEMS
}

\author{
BY MELVYN S. BERGER
}

Communicated by F. John, June 28, 1965

Nonlinear elliptic differential equations of order $m$ acting in a space of $m$ dimensions of ten occupy a special position in more general theories. In this paper we shall study one aspect of this situation. The nonlinear problem under consideration will be the variational approach to eigenvalue problems for nonlinear elliptic partial differential equations as developed by the author in [1], [2], [3], N. Levinson [8] and F. E. Browder [4]. We shall study nonlinearities with exponential type growth, thus filling a gap in the earlier work. A special result applicable in this context, is a theorem of F. John and L. Nirenberg [6], which allows an extension of the Sobolev Imbedding Theorem to Orlicz spaces. The author extends hearty thanks to Professors R. Juberg, W. Littman and N. Meyers for helpful suggestions with this work. This research was partially supported by NSF-GP 2280 .

I. Imbedding Soboler spaces in Orlicz spaces. Let $G$ be a bounded domain in $N$-dimensional real Euclidean space $R^{N}$. We consider various classes of real-valued functions defined on $G$ and their integrals with respect to $N$-dimensional Lebesgue measure. The Sobolev space $W_{m, p}(G)$ consists of all function $u(x)$ in $L_{p}(G)$, whose derivatives (in the sense of L. Schwartz) up to order $m$ are also in $L_{p}(G)$. $w_{m, p}(G)$ is the closure in $W_{m, p}(G)$ of $C_{0}^{\infty}(G) . W_{p}^{\infty}(G)$ is a Banach space with respect to the norm

$$
\|u\|_{m, p}^{p}=\sum_{|\alpha| \leq m}\left\|D^{\alpha} u\right\|_{0, p}^{p} .
$$

Suppose $\phi(t)$ is a real-valued, continuous, convex even function of a real variable such that $\lim _{t \rightarrow 0} \phi(t) / t=0$ and $\lim _{t \rightarrow \infty} \phi(t) / t=\infty$. Then the Orlicz class $L_{\phi}(G)$ with respect to the function $\phi(t)$ consists of all functions $u(x)$ such that $\int_{a} \phi(u(x)) d x<\infty$. An Orlicz class can be made into a Banach space $L_{\phi^{*}}(G)$ by associating with $\phi(t)$ its complementary function $\theta(t)$ and defining

$$
\|u\|=\sup _{v} \int_{G} u v d x, \text { where } \int_{G} \theta(v) d x \leqq 1 .
$$


(Functions, differing only on a set of measure 0 , are considered equivalent.) The closure of the bounded functions in $L_{\phi^{*}}(G)$ will be denoted $E_{\phi}(G)$. If $\phi(t)$ is a function of exponential growth, $E_{\phi}(G)$ $\subset L_{\phi}(G) \subset L_{\phi^{*}}(G)$ are proper set inclusions (Krasnoselskii-Rutickii [7]). A sequence of functions $u_{n}(x)$ is mean convergent to $u(x)$ with respect to the class $L_{\phi}(G)$ if $\int_{G} \phi\left(u_{n}-u\right) \rightarrow 0$ as $n \rightarrow \infty$.

A real-valued function $u(x)$ defined on $G$ is said to have bounded mean oscillation on a bounded cube $K_{0}$, if $\mu(x)$ is integrable over $K_{0}$ and there are constants $c$ and $a_{k}$ such that for every parallel subcube $K$ of edge length $R$, the following inequality holds

$$
\int_{\mathbf{K}}\left|u(x)-a_{k}\right| d x \leqq c R^{N} .
$$

Suppose $X$ and $X_{1}$ are two topological spaces, then $X$ is imbedded continuously in $X_{1}$ if the imbedding operator $i, i(y)=y$ in a continuous one-to-one mapping from $X$ to $X_{1}$. If $i$ is a compact mapping we say the imbedding is compact.

A result of John-Nirenberg [6] shows that if $u(x)$ is of bounded mean oscillation in $C_{0}$, then $u(x) \in L_{\phi}\left(C_{0}\right)$ for any function $\phi(t)$ $\leqq\left[\exp \left(b^{\prime} t\right)-1\right]$ for some $b^{\prime}>0$. It is also known that when $N=m p$ any function $u(x) \in W_{m, p}(G)$ belongs to $L_{p}(G)$ for any $1 \leqq p<\infty$, but $u(x)$ is not necessarily in $L_{\infty}(G)$. The following theorem makes this point precise (cf. Dubinski [5]).

Theorem 1 (An Imbedding Theorem). Let $G$ be a bounded domain in $R^{N}$, and suppose $N=m p$. Then $W_{m, p}(G)$ can be continuously imbedded in $L_{\phi}(G)$ [in the sense of mean convergence] for any $\phi(t)$ $\leqq \exp \left(b^{\prime} t\right)-1\left(\right.$ for some $\left.b^{\prime}>0\right)$. If $\phi(t)=\int_{0}^{t} f(x) d x$ and $\lim _{t \rightarrow \infty}(t / f(\log t))$ $=\infty$, the imbedding of $W_{m, p}(G)$ into $L_{\phi^{*}}(G)$ is continuous and compact.

Proof. Let $K(G)$ be any bounded cube containing $G$ in $R^{N}$. Extend the functions in $W_{m, p}(G)$ to $W_{m, p}(K(G))$ by setting any function $u(x) \in w_{m, p}(G)$ identically equal to zero outside $G$. We now show $u(x)$ regarded as an element in $\varpi_{m, p}\left(K_{0}(G)\right)$ is of bounded mean oscillation on $K_{0}(G)$. First, by the Sobolev Imbedding Theorem if $u(x) \in W_{p}^{m}\left(K_{0}(G)\right),|\operatorname{grad} u(x)| \in L_{N}\left(K_{0}(G)\right)$. Thus modifying Lemma 6 of Serrin [11] we obtain

$$
\int_{\mathbf{K}}\left|u(x)-u_{k}\right| d x \leqq c_{N} R \int_{\mathbf{E}}|\operatorname{grad} u(x)| d x
$$

where $K$ is any subcube parallel to $K_{0}(G)$ with edge length $R, c_{N}$ is a constant depending only on $N$, and $u_{k}=R^{-N} \int_{K} u d x$. Applying Hölder's inequality to (2) we obtain, as in Meyers [10], 


$$
\int_{\boldsymbol{K}(G)}\left|u(x)-u_{k}\right| d x \leqq c_{N} R^{N}\||\operatorname{grad} u(x)|\|_{L_{N}(\boldsymbol{K}(\sigma))} .
$$

Thus $u(x)$ is a function of bounded mean oscillation with respect to $K(G)$ and by the John-Nirenberg Theorem mentioned above $u(x)$ $\in L_{\phi}(G)$ where $\phi(t) \leqq \exp \left(b^{\prime} t\right)-1$ for some $b^{\prime}>0$.

We next demonstrate the continuity of imbedding of $W_{m, p}(G)$ into $L_{\phi}(G)$. Let $u_{n}(x)$ be a sequence of functions converging strongly to $u(x)$ in $W_{m, p}(G)$. Then by the Sobolev Imbedding Theorem $\left|\operatorname{grad} u_{n}\right|$ $\rightarrow|\operatorname{grad} u|$ strongly in $L_{N}(G)$. Thus by equation (3) of [6]

$$
\int_{G}\left[\exp \left(b^{\prime}\left|u-u_{n}\right|\right)-1\right] d x \leqq K_{N}\left\|\left|\operatorname{grad}\left(u_{n}-u\right)\right|\right\|_{0, N} .
$$

Hence $u_{n} \rightarrow u$ in $L_{\phi}(G)$ with respect to mean convergence.

Finally we demonstrate the compactness of the imbedding $\varpi_{m, p}(G)$ $\rightarrow L_{\phi^{*}}(G)$ with $\phi(t)=\int_{0}^{t} f(s) d s$ and $\lim _{t \rightarrow \infty}(t / f(\log t))=\infty$. To this end we use results of Krasnoselskii-Rutickii [7] which imply that a set of functions $X$ in $E_{\phi}$ is compact if $X$ is compact in the sense of convergence in measure, and for some Orlicz function $\phi_{1}(t), \int_{G} \phi_{1}(u) d x$ is uniformly bounded for $u(x) \in X$, where the function $\phi_{1}(t)$ has the property that for all $\lambda>0, \lim _{t \rightarrow \infty}\left(\phi_{1}(\lambda t) / \phi(t)\right)=\infty$. We choose $\phi_{1}(t)=\exp \left(b^{\prime} t\right)$ -1 and by the continuity of the imbedding of $W_{m, p}(G)$ into $L_{\phi_{1}}(G)$ we conclude that if $X$ is a bounded set in $\mathscr{W}_{m, p}(G), \int_{G} \phi_{1}(u) d x$ is uniformly bounded for $u \in X$. Furthermore $u \in X$ implies $\lambda u(x) \in L_{\phi}(G)$ for all $\lambda>0$ thus $u(x) \in E_{\phi}(G)$ where $\phi(t)=\int_{0}^{t} f(s) d s$. Finally an application of Rellich's Lemma shows that $X$ is compact in $L_{2}(G)$ and thus a fortiori with respect to convergence in measure.

II. A limit case for nonlinear elliptic eigenvalue problems. In [8], N. Levinson studied the quasi-linear eigenvalue problem:

$$
\begin{aligned}
\Delta u+\lambda f(u) & =0, \\
\left.u\right|_{\partial G} & =0,
\end{aligned}
$$

where $G$ is a bounded domain in $R^{2}$ subject to the growth assumption

(4) $f(t) \log f(t) \leqq K \int_{0}^{t} f(s) d s$ for some $K \geqq 1$ and $t>0$.

In [1], [2] and [4] Levinson's results are extended to eigenvalue problems for nonlinear elliptic partial differential equations of order $2 m$, of the form $A u=\lambda B u$, acting on bounded domains in $R^{N}$. However an exponential growth assumption analogous to (4) was not obtained. In this section we apply Theorem 1 to determine such a growth assumption for $A$ linear and $N=2 m$. 
Using the definitions and notation of [1], we consider the boundary value problem

$$
\begin{aligned}
A u-\lambda f(u, x) & =0, \\
\left.D^{\alpha} u\right|_{\partial G} & =0, \quad 0 \leqq|\alpha| \leqq m-1,
\end{aligned}
$$

where $A$ is a formally selfadjoint, uniformly elliptic real linear operator of order $2 m$ that satisfies Garding's inequality. We assume $f(t, x) \in Z\left(\rho_{1}\right)$, i.e. $f(t, x)$ is a real-valued function defined on $R^{\prime} \times G$, jointly continuous in the $x$ and $t$ variables, odd and nondecreasing as a function of $t$ and bounded above zero for $x \in G$ and $t>0$ with the exponential condition of growth

$$
\lim _{t \rightarrow \infty} \frac{t}{f(\log t)}=\infty .
$$

(We note that the above growth condition is independent of $m$ and N.)

Set $F(t, x)=\int_{0}^{t} f(s, x) d s$, we then define

$$
\partial M_{R}=\left\{u \mid u \in \mathscr{W}_{m, 2}(G), \int_{G} F(u, x)=R\right\} .
$$

Due to growth assumption (6), we can apply Theorem 1 to conclude that $\partial M_{R}$ is weakly closed in $\mathscr{W}_{m, 2}(G)$.

TheOREM II (EXISTENCE THEOREM). Let $G$ be any bounded domain in $R^{N}$. Suppose $N=2 m$ and $f(t, x) \in Z\left(\rho_{1}\right)$ with the polynomial growth condition replaced by the exponential growth condition (6). Then the boundary value problem (5) has an eigenfunction $u(x)$ [in the generalized sense ]; $u(x)$ is normalized by the requirement that $u(x) \in \partial M_{R}$ for some fixed positive number $R$ and characterized as a solution of the variational problem inf $a(v, v)$ for $v \in \partial M_{R}$.

As the proof of this result is a repetition of an analogous theorem of [1] and [2] (using Theorem 1 in conjunction with the Sobolev Imbedding Theorem), we shall omit it here.

REMARK. If $A$ is a nonlinear operator acting in $w_{m, p}(G)$, as in [4], Theorem 1 can be used to give an exponential growth condition for nonlinear eigenvalue problems of the form $A u=\lambda B u$ for domains $G$ in $R^{N}$ with $N=m p$.

III. A counterexample. The following example emphasizes the importance of the variational approach in the theory of nonlinear eigenvalue problems of the form $A u=\lambda B u$. Consider the two-point boundary value problem (of "Navier-Stokes type") 


$$
\begin{aligned}
\frac{d^{2} u}{d t^{2}}+\lambda u \frac{d u}{d t} & =0, \\
u(a) & =u(b)=0, \quad(-\infty<a<b<\infty) .
\end{aligned}
$$

By direct integration it can be easily shown that (7) has no nontrivial solution in $w_{2}^{1}[a, b]$ for any $\lambda$. However (7) cannot be formulated as a variational problem of Euler-Lagrange type as in Theorem 2.

\section{BIBLIOGRAPHY}

1. M. Berger, An eigenvalue problem for quasi-linear elliptic partial differential equations. Bull. Amer. Math. Soc. 71 (1965), 171-175.

2. - An eigenvalue problem for nonlinear elliptic partial differential equations. Trans. Amer. Math. Soc. 120 (1965), 145-184.

3. - A Sturm-Liouville theorem for non-linear elliptic partial differential equations, Proc. Nat. Acad. Sci. U.S.A. 53 (1965), 1277-1279.

4. F. E. Browder, Variational methods for nonlinear elliptic eigenvalue problems, Bull. Amer. Math. Soc. 71 (1965), 176-183.

5. J. A. Dubinskii, Some imbedding theorems in Orlicz spaces, Dokl. Akad. Nauk. SSSR 152 (1963), 529-532 = Soviet Math. Dokl. 4 (1963), 1339-1342.

6. F. John and L. Nirenberg, On functions of bounded mean oscillation, Comm. Pure Appl. Math. 14 (1961), 415-426.

7. M. A. Krasnoselskii and Y. Rutickii, Convex functions and Orlicz spaces, Noordhoff, Groningen, 1961.

8. N. Levinson, Positive eigenfunctions for $\Delta u+\lambda f(u)=0$, Arch. Rational Mech. Anal. 11 (1962), 258-272.

9. N. Meyers, Mean oscillation over cubes and Hölder continuity, Proc. Amer. Math Soc. 15 (1964), 717-721.

10. J. Serrin, Local behaviour of solutions of quasi-linear equations, Acta. Math. 111 (1964), 247-302.

UNIVERSITY OF MiNNESOTA 